\title{
Spectrum of lung perfusion changes on dual-energy CT in COVID-19: incremental benefit to conventional CT
}

\author{
Srujana Ganti ${ }^{1}$ (D) Shawn Shi Xian Kok ${ }^{1} \cdot$ Syed Aftab ${ }^{1}$ - Sandeep Venkatesh Halagatti ${ }^{1}$ - Steven Bak Siew Wong ${ }^{1}$. \\ Anandswaroop Srinivas Uppaluri ${ }^{1}$
}

Received: 5 September 2020 / Accepted: 27 November 2020 / Published online: 8 January 2021

(C) American Society of Emergency Radiology 2021

\begin{abstract}
The purpose of this case report is to demonstrate lung perfusion changes on dual-energy CT (DECT) in patients with Coronavirus disease 2019 (COVID-19). Since the first case of COVID-19 was reported in Wuhan, Hubei province in China, the spectrum of lung parenchymal findings has been well described but the underlying pathophysiology is less well understood. DECT imaging contributes to the growing evidence that vascular dysregulation has an important role in the underlying pathophysiology of the disease. Three patients with reverse transcriptase polymerase chain reaction (RT-PCR)-confirmed COVID-19 underwent DECT scans. One patient had a DECT for persistent spikes in temperature while the other two patients underwent dual-energy CT pulmonary angiograms (CTPA) for worsening shortness of breath, elevated D dimers and suspected pulmonary embolism. The perfusion abnormalities include focal areas of both hyperperfusion, hypoperfusion, and areas of hypoperfusion surrounded by hyperemia. In addition, dilatation of segmental and subsegmental pulmonary arteries was seen in relation to the lung parenchymal change. DECT has proven useful in supporting the hypothesis that vascular dysregulation plays a significant role in the pulmonary pathophysiology of COVID-19. Early identification and a high index of suspicion is required in the emergency department setting to identify and isolate cases even prior to the results of RT-PCR test being available. Vascular changes on DECT may be an additional radiological feature in detecting the presence of and predicting the severity of disease in the emergency department or acute care setting.
\end{abstract}

Keywords COVID-19 $\cdot$ Lung perfusion · Dual-energy CT, DECT

\section{Introduction}

Since it was first described in Wuhan, Hubei, China, in December 2019, Coronavirus disease 2019 (COVID-19) has proven to be highly contagious, spreading worldwide rapidly with varying rates of morbidity and mortality across different health systems. It was declared a pandemic by the WHO in March 2020 with more than 25 million confirmed cases and 800,000 deaths recorded by August 2020 [1].

The lungs are predominantly afflicted and classical parenchymal changes described include bilateral areas of ground glass change and consolidation in a peripheral or posterior

Srujana Ganti

ganti.srujana@singhealth.com.sg

1 Department of Radiology, Sengkang General Hospital, 110, Sengkang East Way, Singapore 544886, Republic of Singapore distribution [2]. There is increasing evidence supporting the role of a vascular pathology in the underlying pathophysiology. In cases of alveolar insult and hypoxia, the intrinsic physiological response unique to pulmonary arteries is vasoconstriction, such that oxygenated blood is preferentially diverted to better-ventilated areas of the lung, in an attempt to reduce ventilation perfusion mismatch [3]. There is growing recognition that there is dysregulation of this mechanism in patients with COVID-19 with varied vascular changes including dilatation of pulmonary vessels in areas of parenchymal abnormality $[4,5]$. There are many cases of acute pulmonary embolism in patients with COVID-19 pneumonia and its incidence probably underestimated. Emboli are predominantly found in the lobar and segmental vessels, likely precipitated by a combination of factors including an inflammatory prothrombotic state caused by cellular dysfunction $[6,7]$.

Conventional radiographic and computed tomography (CT) findings of lung parenchymal changes in COVID-19 pneumonia have been well described but there is limited 
literature on the use of dual-energy CT (DECT) to illustrate lung perfusion changes. In this case report, we hope to illustrate our experience with three cases of COVID-19 pneumonia demonstrating lung perfusion changes. Post-contrast imaging was acquired as one patient underwent a $\mathrm{CT}$ of the thorax, abdomen, and pelvis (CT TAP) to identify another source of sepsis in addition to evaluating lung parenchymal change. Two patients underwent CT pulmonary angiograms (CTPA) for pulmonary embolism, on a background of COIVD-19 pneumonia, hence necessitating the use of contrast.

\section{Materials and methods}

All three patients underwent DECT utilizing GSI Xtream on on a Revolution CT scanner (GE Healthcare, Waukesha, WI, USA); the voltage was 80 to $120 \mathrm{kV}$, matrix $512 \times 512$, and slice thickness $3 \mathrm{~mm}$. Contrast for the CT TAP was injected at a rate of $1.8 \mathrm{ml} / \mathrm{sand}$ at a rate of $3.6 \mathrm{ml} / \mathrm{scontrast}$ for the CTPA. Data was transferred to AW server 3.2 and analyzed with Gemstone Spectral Imaging (GSI) Pulmonary Perfusion Software. The scans were reviewed by two radiologists specializing in body imaging.

\section{Cases}

\section{Case 1}

A 35-year-old male patient was admitted with COVID-19 pneumonia, confirmed on nasopharyngeal swab RT-PCR. $\mathrm{He}$ was experiencing persistent spikes of fever $\left(>38.0^{\circ} \mathrm{C}\right)$ at day 12 of illness without any other localizing cause of infection. He was not tachypneic and did not require supplemental oxygen. CT TAP was performed to evaluate the severity of lung disease and exclude other causes of sepsis (Figs. 1 and 2).

\section{Case 2}

A 34-year-old male patient was diagnosed with COVID-19 infection on routine nasopharyngeal RT-PCR swab. He experienced persistent fever, worsening shortness of breath, and chest pain and was admitted to our institution on day 2 of illness. Chest radiograph performed in the emergency department showed bilateral lower zone airspace opacities. Arterial blood gas findings were suggestive of type 1 hypoxic respiratory failure and he was admitted to the intensive care unit. Ddimer quantitation was elevated at $0.86 \mathrm{mg} / \mathrm{l}$ fibrinogenequivalent units (FEU) (normal range: $0.19-0.55 \mathrm{mg} / \mathrm{l} \mathrm{FEU}$ ). He underwent a CTPA for suspected pulmonary embolism (Fig. 3a-f).

\section{Case 3}

A 67-year-old male patient was admitted for COVID-19 pneumonia on day 2 of illness. On day 9 of illness, he developed worsening shortness of breath requiring increasing oxygen supplementation with chest radiographs showing progression of bilateral pneumonia. He was transferred to the intensive care unit and was subsequently intubated. D-dimer levels were considerably elevated at $17.09 \mathrm{mg} / \mathrm{FEU}$ (normal range $0.19-0.55 \mathrm{mg} /$ 1 FEU). DECT pulmonary angiogram was performed on day 14due to increasing hypoxia and ventilator requirements, but was negative for pulmonary embolism (Fig. 4).

\section{Discussion}

The spectrum of lung parenchymal changes in COVID-19 demonstrates a fairly typical pattern. Pan et al. described a temporal relationship of CT chest findings, characterized by 4 stages of progression [8]. The initial findings include predominantly lower lobe subpleural ground glass change (stage 1); bilateral multilobar ground glass change and consolidation (stage 2); dense consolidation, crazy paving pattern, and

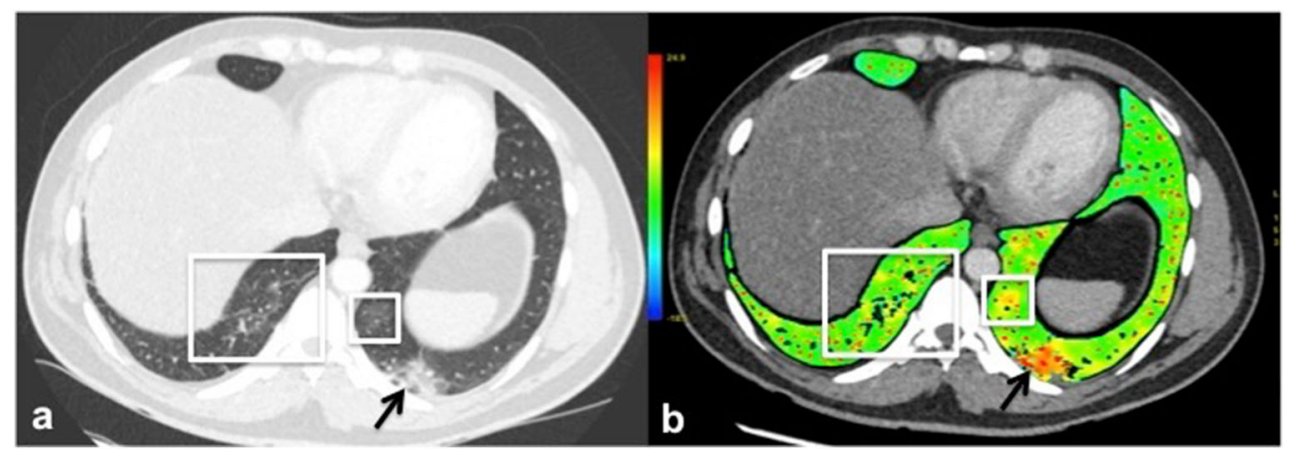

Fig. 1 a Axial image of the lung bases show a focal consolidation with peripheral ground glass opacity in the posterobasal left lower lobe (black arrow). Subtle ground glass opacities are also visible more medially in the left lower lobe and the basal right lower lobe (white frames). b
Corresponding perfusion blood volume (PBV) image shows considerably increased perfusion in the left lower lobe (black arrow). A few areas of mild hypervascularity are also recognizable in the rest of the lung bases with subtle ground glass change (white frames) 
Fig. 2 a, b Axial image of the upper lungs and corresponding maximum intensity projection (MIP) image demonstrate scattered ground glass opacities bilaterally (white frames), with abnormally dilated subsegmental vessels in some of the central and peripheral opacities at contiguous slices (white arrows). c PBV image at the same level shows regional areas of increased perfusion corresponding to the ground glass opacities (white frames). d Corresponding iodine map image also demonstrates hyperperfusion as well as segmental and subsegmental vasodilatation, especially at the medial aspect of right upper lobe and the apical segment of the left lower lobe (white frames)
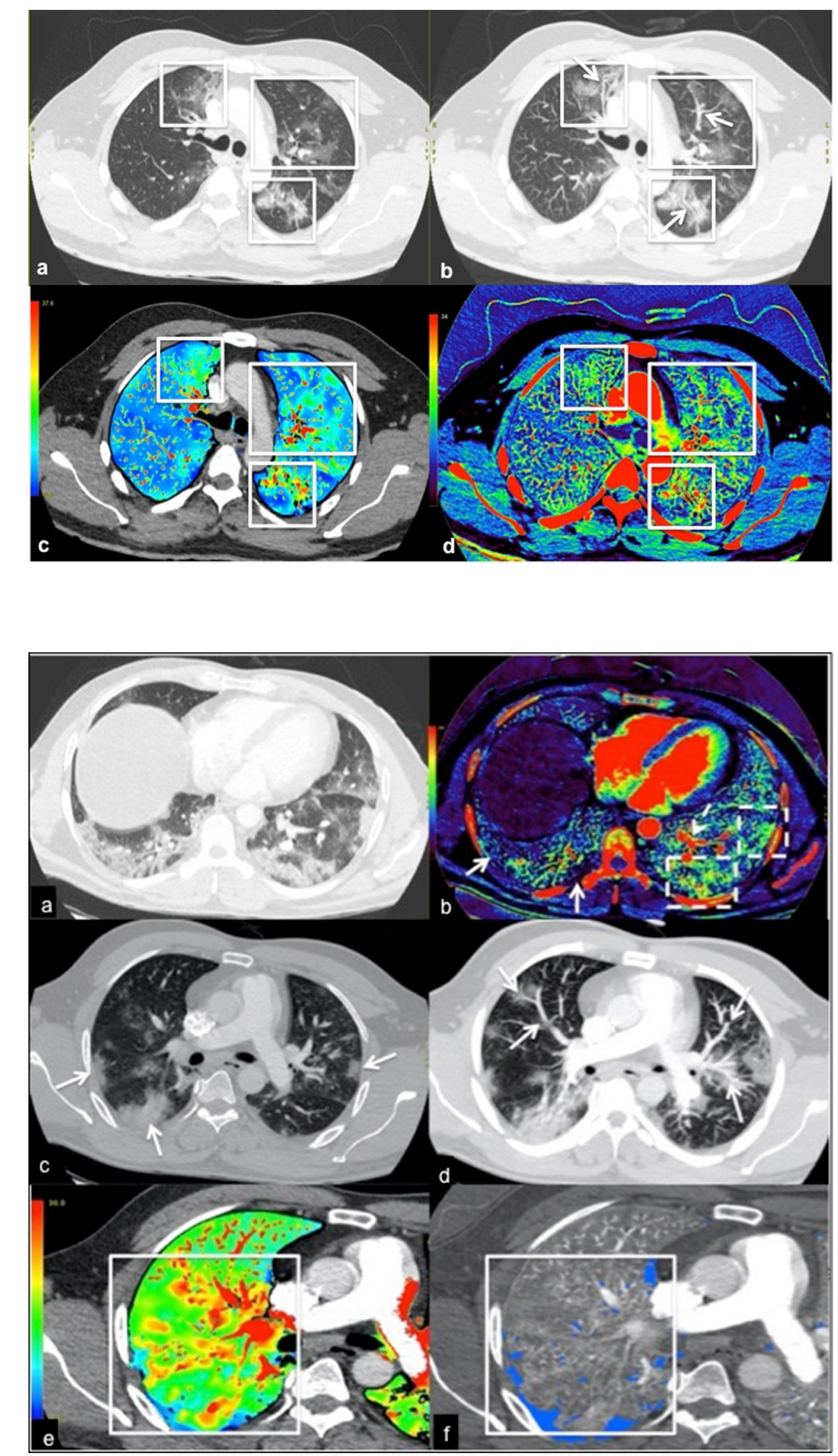

Fig. 3 a CT pulmonary angiogram axial image at the lung base demonstrates extensive peripheral and subpleural opacities. b Corresponding iodine image shows areas of hyperperfusion in some of the opacities in the left lower lobe (dashed white boxes), while some areas show normal to reduced perfusion in some of the opacities in the right lower lobe (solid white arrows). Note also the dilated segmental pulmonary arteries in the left lower lobe (dashed white arrow). c Axial image of the upper lungs showing a few peripheral subpleural opacities (white arrows). d Axial MIP image demonstrating segmental and subsegmental vessel enlargement (white arrows). e, $\mathbf{f}$

Corresponding lung PBV images show subpleural areas of perfusion defects in the right lung, which are associated with areas of increased perfusion proximally (white boxes). No pulmonary embolism was identified 
Fig. 4 a Axial image from CT pulmonary angiogram showing dense consolidation in the left lung (white frame) and ground glass opacities in the right lung (black arrows). b Corresponding iodine image shows a perfusion defect within the left lung with a surrounding halo of increased perfusion (white frame). No pulmonary embolism was detected

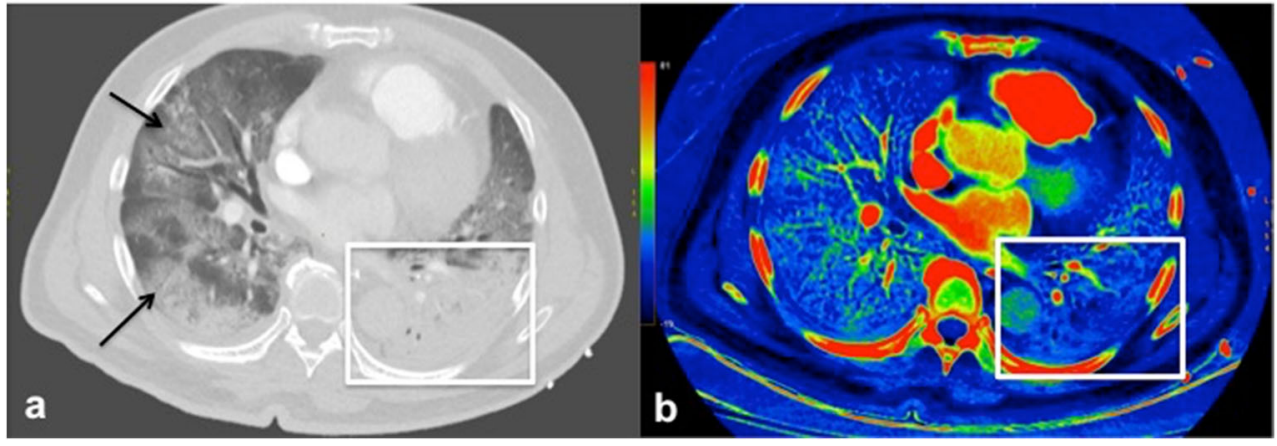

parenchymal bands (stage 3); and final stage of resolution with the absorption of consolidation and lack of visualization of the crazy paving pattern (stage 4) [8]. We can propose that patients 1 and 2 were in stage 2 of the disease while patient 3 was in stage 3 of the disease.

DECT works on the principle of utilizing two different x-ray energy spectra (high- and low-kilovoltage series) during image acquisitions. By using various post processing techniques, different sequences such as virtual non-contrast, iodine images and lung perfusion maps can be generated. Hence, DECT with added post-processing allows the radiologist to assess for perfusion abnormalities in addition to the lung parenchyma.

The cases above, although negative for pulmonary embolism, demonstrated a spectrum of lung perfusion changes ranging from hypoperfusion to hyperperfusion. We noted dilatation of blood vessels in the subpleural region and within the affected lungs in all three patients, in keeping with previously published findings. Caruso et al. describe finding enlarged subpleural blood vessels (>3 mm) in $89 \%$ of their patients [9]. This is corroborated by the study by Lang et al. who found dilated pulmonary vasculature in $85 \%$ of cases and dilated distal blood vessels extending to the pleura and fissures [4]. Alberello et al. described two cases in which there was a progressive increase in pulmonary artery caliber and pulmonary vessel hypertrophy in areas of increased ground glass opacification, suggesting that it could be an early radiological sign to predict lung deterioration [5]. The proposed mechanisms to explain this underlying change include abnormal vasoregulation, intrapulmonary shunting, or microvascular thrombosis. Ackermann et al. described severe endothelial damage within the lungs in a small group of seven patients with COVID-19 and a process of angiogenesis accounting for new vessel growth, much more evident in COVID-19 patients than in influenza, which may account for the vessel dilatation noted on CT [10]. Recent literature has also shed light on the seemingly "happy" hypoxic patient who, though fairly hypoxemic based on physiological parameters, might not initially experience significant symptomatic dyspnea due to preserve lung compliance but subsequently experiences rapid deterioration [11].

The reporting time for COVID-19 swab results is variable, dependent on local policy and resource availability. There have been multiple cases in which the initial COVID-19 RT-
PCR result has been false negative with concurrent CT demonstrating positive lung parenchymal changes [12]. The CT findings of ground glass opacities and peripheral consolidation are not specific and may be seen in a variety of diseases. DECT could strengthen diagnostic confidence over conventional CT in circumstances where the initial COVID swab is negative or pending, thereby aiding clinical management.

The limitations of the study are that these are preliminary results in a small number of cases from a single institution. Further analysis on a larger cohort of cases with varying severity of lung parenchymal involvement would be of value. It remains a work in progress to determine if early identification of areas of perfusion anomalies on DECT in the "happy" hypoxic patient who is compensating well could be a useful prognostic indicator for predicting deterioration. If validated, the routine use of DECT (if available) may be helpful in assessing for perfusion anomalies, a feature which could even be incorporated as part of a COVID-19 pneumonia CT severity scoring system.

\section{Conclusion}

Our findings support the emerging literature that pulmonary vascular dysregulation has a significant role in the pathogenesis of COVID-19-related lung disease. Utilizing DECT gives additional insight into the disease pathophysiology by assessing lung perfusion changes. Early identification of vascular changes may be a harbinger of further deterioration and could influence the decision-making algorithm for emergency clinicians, including isolation of patients and clinical management; however, further research is still required.

Code availability Not applicable.

Data availability Not applicable.

\section{Compliance with ethical standards}

Conflict of interest The authors declare that they have no conflict of interest. 
Informed consent Informed consent from patients was waived.

Consent for publication All authors give consent for publication.

\section{References}

1. Coronavirus disease (COVID-19) Weekly Epidemiological Update 31 August 2020 - https:/www.who.int/docs/default-source/ coronaviruse/situation-reports/20200831-weekly-epi-update-3. pdf?sfvrsn=d7032a2a_4[Accessed on September 4, 2020]

2. Bernheim A, Mei X, Huang M, Yang Y, Fayad ZA, Zhang N, Diao K, Lin B, Zhu X, Li K, Li S, Shan H, Jacobi A, Chung M (2020) Chest CT findings in coronavirus disease-19 (COVID-19): relationship to duration of infection. Radiology 295(3):200463. https://doi. org/10.1148/radiol.2020200463

3. Dunham-Snary KJ, Wu D, Sykes EA, Thakrar A, Parlow LRG, Mewburn JD, Parlow JL, Archer SL (2017) Hypoxic pulmonary vasoconstriction: from molecular mechanisms to medicine. Chest 151(1):181-192. https://doi.org/10.1016/j.chest.2016.09.001

4. Lang M, Som A, Carey D, Reid N, Mendoza DP, Flores EJ, Li MD, Shepard J-AO, Little BP (2020) Pulmonary vascular manifestations of COVID-19 pneumonia. Radiology 2(3):e200277. https://doi.org/ 10.1148/ryct.2020200277

5. Albarello F, Pianura E, Di Stefano F, Cristofaro M, Petrone A, Marchioni L, Palazzolo C, Schinina V, Nicastri E, Petrosillo N, Campioni P, Eskild P, Zumla A, Ippolito G, Group CIS (2020) 2019-novel coronavirus severe adult respiratory distress syndrome in two cases in Italy: an uncommon radiological presentation. Int $\mathrm{J}$ Infect Dis 93:192-197. https://doi.org/10.1016/j.ijid.2020.02.043
6. Cellina M, Oliva G (2020) Acute pulmonary embolism in a patient with COVID-19 pneumonia. Diagn Interv Imaging 101(5):325326. https://doi.org/10.1016/j.diii.2020.04.001

7. Roncon, L., Zuin, M., Barco, S., Valerio, L., Zuliani, G., Zonzin, P., \& Konstantinides, S. V. (2020). Incidence of acute pulmonary embolism in COVID-19 patients: systematic review and meta-analysis. Eur J Intern Med, S0953-6205(20)30349-6. Advance online publication. https://doi.org/10.1016/j.ejim.2020.09.006

8. Pan F, Ye T, Sun P, Gui S, Liang B, Li L, Zheng D, Wang J, Hesketh RL, Yang L, Zheng C (2020) Time course of lung changes at chest CT during recovery from coronavirus disease 2019 (COVID-19). Radiology 295(3):715-721. https://doi.org/10.1148/ radiol.2020200370

9. Caruso D, Zerunian M, Polici M, Pucciarelli F, Polidori T, Rucci C, Guido G, Bracci B, De Dominicis C, Laghi A (2020) Chest CT features of COVID-19 in Rome, Italy. Radiology 296(2):E79-E85. https://doi.org/10.1148/radiol.2020201237

10. Ackermann M, Verleden SE, Kuehnel M, Haverich A, Welte T, Laenger F, Vanstapel A, Werlein C, Stark H, Tzankov A, Li WW, Li VW, Mentzer SJ, Jonigk D (2020) Pulmonary vascular endothelialitis, thrombosis, and angiogenesis in Covid-19. N Engl J Med 383(2):120-128. https://doi.org/10.1056/NEJMoa2015432

11. Dhont S, Derom E, Van Braeckel E, Depuydt P, Lambrecht BN (2020) The pathophysiology of 'happy' hypoxemia in COVID-19. Respir Res 21(1):198. https://doi.org/10.1186/s12931-020-01462-5

12. Xie X, Zhong Z, Zhao W, Zheng C, Wang F, Liu J (2020) Chest CT for typical coronavirus disease 2019 (COVID-19) pneumonia: relationship to negative RT-PCR testing. Radiology 296(2):E41E45. https://doi.org/10.1148/radiol.2020200343

Publisher's note Springer Nature remains neutral with regard to jurisdictional claims in published maps and institutional affiliations. 